\title{
Prevalencia de Trastornos Temporomandibulares según los CDI/TTM, en un Grupo de Adultos Mayores de Santiago, Chile
}

\author{
Prevalence of Temporomandibular Disorders According \\ to RDC/TMD in Older People of Santiago, Chile
}

Isbel Sandoval"; Nicole Ibarra**; Guillermo Flores" ${ }^{* *}$; Katina Marinkovic"; Walter Díaz ${ }^{* * *}$ \& Fernando Romo*

SANDOVAL, I.; IBARRA, N.; FLORES, G.; MARINKOVIC, K.; DÍAZ, W. \& ROMO, F. Prevalencia de trastornos temporomandibulares según los CDI/TTM, en un grupo de adultos mayores de Santiago, Chile. Int. J. Odontostomat., 9(1):73-78, 2015.

RESUMEN: En la población chilena, los adultos mayores representan un 13\% de la población total del país (2,2 millones), proyectándose para el 2020 un aumento del grupo de 60 años o más en un $45 \%$. Todos los seres humanos sufren un proceso de envejecimiento y, los tejidos orales y periorales no escapan de este proceso. Las repercusiones sobre el sistema estomatognático, podrían manifestarse a través de sintomatología en alguno de sus componentes, compatible con un trastorno temporomandibular (TTM). El objetivo de este trabajo, fue determinar la prevalencia de TTM según los Criterios de Investigación Diagnóstica ó CDI/TTM (Eje I), en adultos mayores examinados en la Clínica Odontológica de la Facultad de Odontología de la Universidad de Chile (FOUCh), durante el año 2012 y establecer la relación entre ambos sexos. Este estudio determinó, que existe una alta prevalencia de TTM en la muestra de adultos mayores chilenos estudiados (47\%), principalmente diagnósticos de tipo articular y sin una relación significativa entre ambos sexos.

PALABRAS CLAVE: adultos mayores, trastornos temporomandibulares, CDI/TTM.

\section{INTRODUCCIÓN}

Los Trastornos temporomandibulares (TTM) son un grupo heterogéneo de patologías que afectan la articulación temporomandibular (ATM), músculos masticatorios, o ambos (Griffiths, 1983). Caracterizadas clásicamente por una triada de signos descritos en clínica: dolor muscular o articular; ruidos articulares; y restricción, desviación o deflexión en la apertura mandibular (Laskin, 1969).

La prevalencia real de TTM a nivel poblacional es una cuestión de debate, debido a la falta de homogeneidad en los criterios de diagnóstico que adoptaron las diferentes investigaciones. Hay evidencia de que la prevalencia de signos y síntomas de TTM pueden ser altos en la población general (Dworkin et al., 1990). En forma conservadora se podría estimar que el número de individuos en la población general con algún TTM es del orden del 40-50\%. La mayor prevalencia de síntomas de TTM se ha observado entre los 20 y 40 años de edad, con menor prevalencia en los más jóvenes y las personas mayores (Leresche et al., 2008).

Respecto a la prevalencia según sexo, gran parte de la literatura coincide en que hay una mayor frecuencia de TTM en mujeres que en hombres, con relaciones que van desde 3:1 a 6:1 (Corsini et al., 2005; Phillips et al., 2001).

Las diferencias en los protocolos clínicos utilizados para establecer los diagnósticos de TTM, pueden ser los responsables de la alta variabilidad de los resultados presentes en los diferentes estudios existentes en la literatura. La introducción de los Criterios

* Clínica de Odontología Integral del Adulto, Departamento de Prótesis, Universidad de Chile, Santiago, Chile.

" Área de Oclusión, Traumatología, TTM y DOF, Departamento de Prótesis, Universidad de Chile, Santiago, Chile.

${ }^{* * *}$ Especialista en TTM y DOF, Docente a cargo de la Clínica de Odontología Integral del Adulto, Departamento de Prótesis, Universidad de Chile, Santiago, Chile. 
Diagnósticos de Investigación (CDI/TTM) en 1992 pretendió aumentar el nivel de coherencia entre los estudios, gracias al uso de criterios diagnósticos estandarizados. Estos CDI/TTM, utilizan métodos de examen clínico y obtención de historial con validez científicamente demostrada, para la obtención de signos clínicos de TTM (Eje I), y la evaluación del aspecto conductual, psicológico y psicosocial (Eje II) (Dworkin \& LeResche, 1992).

Por otra parte, el envejecimiento poblacional es un problema mundial. La población en países en vías de desarrollo contribuye de manera significativa a la transformación demográfica mundial. Estas tendencias en los patrones globales de envejecimiento tienen implicaciones importantes para la incidencia actual y futura en la prevalencia de muchas condiciones de salud. El envejecimiento global tiene implicaciones importantes para la prestación de asistencia sanitaria, como los cambios fisiológicos relacionados con la edad se pasan por alto con frecuencia cuando se consideran opciones de tratamiento y pueden dar lugar a resultados adversos innecesarios (Lee et al., 2011). En la población chilena, según el informe del Instituto Nacional de Estadística (INE) del año 2010, desde la década de los años 60 , ha ocurrido un continuo envejecimiento demográfico, en el que los adultos mayores representan un $13 \%$ de la población total del país (2,2 millones). Proyectándose para el 2020 un aumento del grupo de 60 años o más en un 45\% (3,2 millones de habitantes) (INE, 2010).

Todos los seres humanos sufren un proceso de envejecimiento, el que es irreversible, dinámico, progresivo, declinante y heterogéneo. Cada especie tiene sus propias características de envejecimiento y varía de sujeto a sujeto, y de órgano a órgano dentro del mismo individuo. En los humanos ocurren cambios biopsicosociales resultantes de la interacción de factores genéticos, sociales, culturales, del estilo de vida y la presencia de enfermedades que determinan el envejecimiento (Ministerio de la Mujer y Desarrollo Social de Perú, 2003). Afecta a todos los tejidos, órganos y sistemas, y por tanto, en la medida que el individuo tiene más edad, el compromiso sistémico es mayor y más complejo. Los tejidos orales y periorales no escapan de este proceso y es aquí donde se pueden observar diferentes cambios (Levy, 1991; Drummond et al., 1994). Con la edad se van generando cambios funcionales y patológicos en el sistema masticatorio, debido a que existen condicionantes propias de los adultos mayores; ellos son en su mayoría desdentados parciales, o bien desdentados totales (Taboada et al., 2004). En Chile, según la Encuesta Nacional de Salud 2003, los adultos de 65 a 74 años presentan un amplio historial de caries, la prevalencia de desdentados parciales alcanza el $69,8 \%$ y de desdentados totales el 29,1\% (Minsal, 2010). La pérdida dentaria genera falta de soporte posterior y por ende avance mandibular y disminución de la dimensión vertical. Las repercusiones que esto podría tener sobre el cóndilo y disco articular se evidencian principalmente en la presencia de sintomatología dolorosa de la ATM y otros síntomas como ruidos, dolor a la palpación y apertura limitada (Taboada et al.).

Sin embargo, aún existe controversia respecto a la etiología de los TTM, es por ello, que se prefiere hablar de factores de riesgo de los TTM, los cuales no son capaces de provocar enfermedad por sí solos, pero asociados con otros factores, sobrepasan la capacidad homeostática del individuo desencadenando la patología (Okeson, 2003; Pino, 2001). Dependiendo del rol que desempeñen estos factores de riesgo hablaremos de factores predisponentes, precipitantes y perpetuantes (Okeson).

Para determinar cómo influye la oclusión en la posible aparición de un TTM, es necesario entender cómo influye la oclusión en la estabilidad ortopédica del sistema. Existe estabilidad ortopédica cuando la posición intercuspídea estable de los dientes, está en armonía con la posición musculoesquelética estable de los cóndilos en las fosas articulares (Okeson).

Una oclusión estable no debería ocasionar ningún problema, y los cambios funcionales o estructurales se producen dentro de límites fisiológicos; el problema surge cuando existe un sistema masticatorio inestable, que recibe carga de parte de los músculos elevadores, obligando al sistema a realizar movimientos con modificaciones de engramas musculares, para lograr una mayor estabilidad oclusal, llevando todo esto a una inestabilidad articular (Okeson). Es así, como los signos y síntomas generalmente aparecen cuando la alteración supera la tolerancia fisiológica propia de cada individuo, en la región afectada, originando una respuesta alterada en el sistema. El paciente lo describe como una limitación del movimiento mandibular con dolor asociado, ruidos y dolor articular, movilidad o desgaste de los dientes, dolores de cabeza y faciales (Okeson).

En nuestro país y en el mundo, no existen datos suficientes respecto a los trastornos temporomandibulares que puedan estar afectando a 
la población de adultos mayores, lo que no ha permitido al menos en Chile, el desarrollo de políticas públicas de salud, para tales afecciones, ya que los servicios de salud oral, al no poseer los recursos necesarios para toda la población, priorizan la atención en la población de menor edad. A pesar de aquello, un 75,3\% de nuestra población mayor geriátrica hace uso del sistema público de atención en salud (MIDEPLAN, 2012), debido a la mayor prevalencia de enfermedades de carácter crónico-degenerativo (artritis, cáncer, EPOC, diabetes, enfermedades cardíacas, hipertensión, condiciones mentales, osteoporosis, Parkinson e infartos) (Scully \& Ettinger, 2007). Esto se debe, dentro de otros factores, a que en el sistema inmune se manifiestan alteraciones en el sistema de reconocimiento y regulación, con pérdida de la capacidad de respuesta frente a los agentes externos y aumento de los fenómenos autoinmunes (Misrachi et al., 2005). Lo cual significa entonces un desafío para nuestro país, y seguramente para el mundo, que deberá reorientar el servicio público de salud, para satisfacer las necesidades de esta creciente población (Petersen \& Yamamoto, 2005).

En este ámbito, este trabajo ha pretendido establecer una línea de investigación en TTM en adultos mayores, encuadrada en un proyecto mayor que intenta determinar la prevalencia de signos y síntomas de TTM y de los diferentes diagnósticos para TTM tomando en cuenta el sistema estandarizado establecido por los CDI/TTM.

\section{MATERIAL Y MÉTODO}

Se realizó un estudio descriptivo, de corte transversal, en 100 pacientes adultos mayores, elegidos según criterios de inclusión, individuos de 60 años o más; que estuvieran dispuestos a participar de manera voluntaria firmando el consentimiento informado, y criterios de exclusión, establecidos por el protocolo de los CDI/TTM. Los sujetos fueron elegidos, de un uni- verso de 140 pacientes, que se encontraban en tratamiento odontológico activo, en las Clínicas de Prótesis Parcial Removible y Clínica Integral del adulto, durante el año 2012, en la Facultad de Odontología de la Universidad de Chile (FOUCh).

La recolección de la información se realizó, siguiendo el protocolo de examen clínico de los CDI/ TTM (Eje I), por dos alumnas egresadas de la FOUCh, previamente calibradas por un especialista en el tema, hasta obtener un nivel de confianza diagnóstica sobre un $80 \%$. Según los datos recolectados, los pacientes fueron diagnosticados para TTM, según la clasificación de los CDI/TTM presentados en la Tabla I, usando los algoritmos diagnósticos para tales Criterios de Investigación diagnóstica.

Para determinar la prevalencia general de TTM de la población adulta mayor elegida, en el caso de los diagnósticos de TTM de tipo articular e inflamatorio (Tipo II y III, Tabla I), se consideró "individuo enfermo" a aquellos que presentaron al menos una de las articulaciones comprometidas, ya sea la ATM derecha o izquierda. Los diagnósticos de TTM fueron analizados, como variables de prevalencia en forma descriptiva y con diferencia de proporciones, para analizar la diferencia entre los sexos.

\section{RESULTADOS}

Análisis de los Datos Demográficos. La distribución de los sujetos por sexo y edad, se presentan en la Tabla II. El rango de edad de los pacientes examinados fluctuó entre los 60 y 90 años, con un promedio de edad de $67,6 \pm 6$ años, de los cuales el $67 \%$ fueron individuos de sexo femenino $(n=67)$ y el $33 \%$ de sexo masculino $(n=33)$. El mayor número de individuos examinados estuvo en el rango de 60 a 69 años.

Prevalencia de TTM y Subgrupos diagnósticos. La prevalencia de sujetos que presentó al menos un diag-

Tabla I. Clasificación Diagnóstica de Trastornos Temporomandibulares según CDI/TTM.

\begin{tabular}{|c|c|c|}
\hline Grupo I: TTM muscular & Grupo II: TTM articular & Grupo III Inflamatorio \\
\hline Grupo I a: Dolor miofascial & $\begin{array}{l}\text { Grupo II a: Desplazamiento de disco con } \\
\text { reducción. }\end{array}$ & Grupo III a: Artralgía \\
\hline $\begin{array}{l}\text { Grupo I b: Dolor miofascial con } \\
\text { limitaciones de apertura. }\end{array}$ & $\begin{array}{l}\text { Grupo II b: Desplazamiento de disco sin } \\
\text { reducción con limitación de apertura. }\end{array}$ & Grupo III b: Osteoartritis de ATM \\
\hline \multirow[t]{2}{*}{ Grupo I c: Sin Diagnóstico en el grupo I } & $\begin{array}{l}\text { Grupo II c: Desplazamiento de disco sin } \\
\text { reducción con limitación de apertura. }\end{array}$ & Grupo III c: Osteoartritis de ATM \\
\hline & Grupo II d: Sin Diagnóstico en el grupo II & Grupo III d: Sin Diagnóstico en el grupo III \\
\hline
\end{tabular}


SANDOVAL, I.; IBARRA, N.; FLORES, G.; MARINKOVIC, K.; DÍAZ, W. \& ROMO, F. Prevalencia de trastornos temporomandibulares según los CDI/TTM, en un grupo de adultos mayores de Santiago, Chile. Int. J. Odontostomat., 9(1):73-78, 2015.

nóstico de TTM fue de 47\% (Fig. 1). Los resultados al considerar los Grupos diagnósticos según los CDI/TTM se muestran en la Figura 2 y para los subgrupos diagnósticos en la Tabla III. La mayor proporción de sujetos diagnosticados correspondió al Grupo II (33\%), los cuales en su totalidad se encontraron en el subgrupo Ila (desplazamiento discal con reducción). Los del Grupo III presentaron una prevalencia de $13 \%$, representados por los subdiagnósticos de Illa (artralgia) y IIIc (osteoartrosis); el Grupo I fue el menos prevalente en la población estudiada con un $6 \%$ correspondiente al subdiagnóstico la (dolor miofascial sin limitación de apertura).

Prevalencia de TTM según sexo. La prevalencia de trastornos temporomandibulares según sexo, fue de un $52,2 \%$ para las mujeres y de un $36,4 \%$ para los hombres. Al realizar la estimación de diferencia de proporciones, no se observaron diferencias significativas en la prevalencia de TTM por sexo en la muestra estudiada (PRTesti $\mathrm{P}=0,0683$ ).
Tabla II. Distribución de los pacientes según edad y sexo.

\begin{tabular}{lcccccc}
\hline \multirow{2}{*}{ Edad (años) } & \multicolumn{2}{c}{ Mujeres } & \multicolumn{2}{c}{ Hombres } & \multicolumn{2}{c}{ Total } \\
\cline { 2 - 7 } & $\mathrm{n}$ & Prev. (\%) & $\mathrm{n}$ & Prev. (\%) & $\mathrm{n}$ & Prev. (\%) \\
\hline $60-69$ & 41 & $41 \%$ & 24 & $24 \%$ & 65 & $65 \%$ \\
$70-79$ & 23 & $23 \%$ & 8 & $8 \%$ & 31 & $31 \%$ \\
$80-90$ & 3 & $3 \%$ & 1 & $1 \%$ & 4 & $4 \%$ \\
Total & 67 & $67 \%$ & 33 & $33 \%$ & 100 & $100 \%$ \\
\hline
\end{tabular}

La distribución considerando la prevalencia de TTM según los subgrupos diagnósticos de CDI/TTM y sexo, se muestra en la Tabla III. El diagnóstico más prevalente tanto en hombres como en mujeres fue del subgrupo lla (desplazamiento discal con reducción). Por el contrario, el de menor prevalencia, fue el diagnóstico del subgrupo IIIC (osteoartrosis), con un 7,5\% en mujeres y sólo un $5 \%$ en hombres.

Tabla III. Distribución por sexo, según los subgrupos diagnósticos CDI/TTM.

\begin{tabular}{|c|c|c|c|c|c|c|c|}
\hline \multirow[t]{2}{*}{$\begin{array}{l}\text { Diagnóstico } \\
\text { (Grupo) }\end{array}$} & \multicolumn{2}{|c|}{$\begin{array}{c}\text { Mujeres } \\
(n=67)\end{array}$} & \multicolumn{2}{|c|}{$\begin{array}{l}\text { Hombres } \\
(n=33)\end{array}$} & \multicolumn{2}{|c|}{$\begin{array}{c}\text { Total } \\
(n=100)\end{array}$} & \multirow[t]{2}{*}{$\mathrm{P}$} \\
\hline & $\mathrm{n}$ & Prev. (\%) & $\mathrm{n}$ & Prev. (\%) & $\mathrm{n}$ & Prev. (\%) & \\
\hline I a Dolor Miofascial & 6 & 9 & 0 & 0 & 6 & 6 & 0,075 \\
\hline I b Dolor Miofascial con limitación de la apertura & 0 & 0 & 0 & 0 & 0 & 0 & \\
\hline II a Desplazamiento discal con reducción & 21 & 31,3 & 12 & 36,5 & 33 & 33 & 0,61 \\
\hline $\begin{array}{l}\text { II b Desplazamiento de disco sin reducción con } \\
\text { limitación de apertura. }\end{array}$ & 0 & 0 & 0 & 0 & 0 & 0 & \\
\hline $\begin{array}{l}\text { Il c Desplazamiento de disco sin reducción sin } \\
\text { limitación de apertura. }\end{array}$ & 0 & 0 & 0 & 0 & 0 & 0 & \\
\hline III a Artralgia & 8 & 11,9 & 0 & 0 & 8 & 8 & 0,551 \\
\hline III b Osteoartritis & 0 & 0 & 0 & 0 & 0 & 0 & \\
\hline III c Osteoartrosis & 5 & 7,5 & 0 & 0 & 5 & 5 & 0,637 \\
\hline
\end{tabular}

$\mathrm{P}=$ prueba de diferencia de proporciones para sexo significativo $<0,05$.

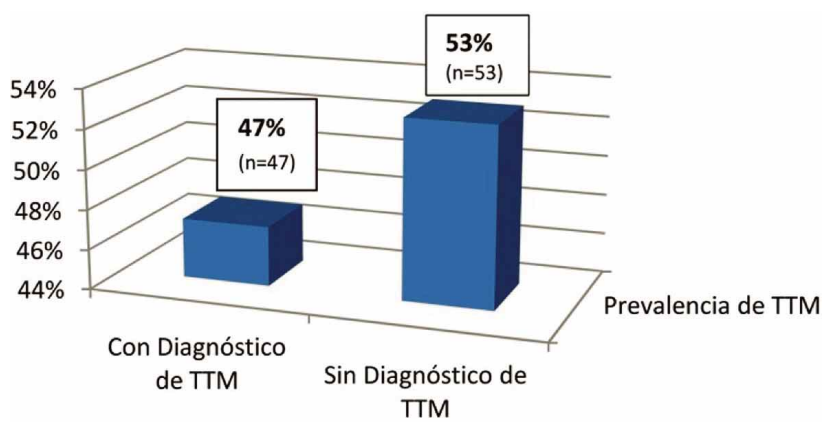

Fig. 1. Prevalencia General de TTM en la muestra $(n=100)$.

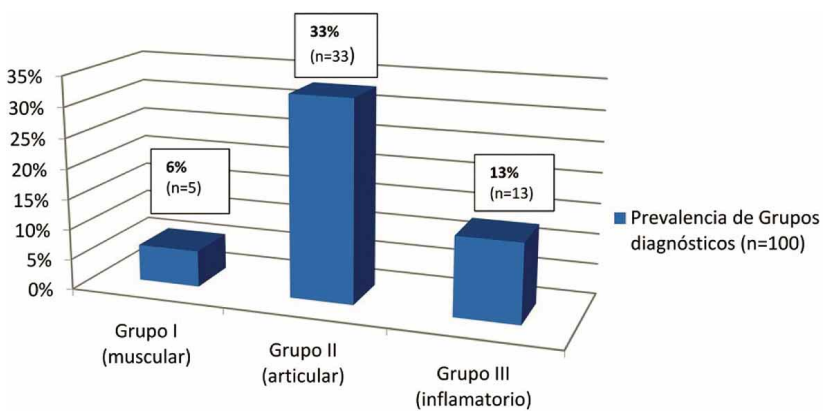

Fig. 2. Prevalencia de Grupos diagnósticos según los CDI/ TTM. 


\section{DISCUSIÓN}

Los escasos estudios previos de prevalencia de TTM en el adulto mayor no han utilizado un protocolo de examen estandarizado y validado. Es por esta razón que la prevalencia de tales patologías en este grupo etario aún poco clara. El objetivo general de este estudio fue determinar la prevalencia general de TTM en adultos mayores, diagnosticados según los CDI/ TTM (Dworkin \& LeResche), en los pacientes atendidos en la Clínica Odontológica de la FOUCh, en las áreas de Prótesis Removible e Integral del Adulto, durante el año 2012.

Las prevalencias obtenidas, según grupos diagnósticos (muscular, articular o inflamatorio) o alguna asociación entre ellos, concuerda con lo descrito por otros autores, que indican que los TTM son de alta prevalencia en la población. Los TTM son de muy baja prevalencia en la niñez, por otra parte aumentan durante el periodo adulto y posteriormente disminuyen en la vejez. En estudios previos, se ha determinado que la mayor prevalencia de TTM se encuentra en el rango de 30 a 45 años (Yap et al., 2003). Adicionalmente, algunos estudios de manera conservadora estiman que el número de individuos con algún tipo de TTM varia entre un 40 a un $60 \%$ (Grau et al., 2005), principalmente en individuos entre 20 a 40 años y de sexo femenino (Dworkin et al.; Grau et al.).

Respecto a la distribución de TTM según sexo, en este estudio, se observó un mayor porcentaje de mujeres $(52,2 \%)$, con algún diagnóstico de TTM, en comparación con los hombres $(36,4 \%)$, aunque esta diferencia no fue estadísticamente significativa, lo que en alguna medida podría deberse a una muestra insuficiente. Sin embargo, la mayor prevalencia de TTM en mujeres, fue coincidente con lo reportado en la lite- ratura donde este grupo en términos generales, sufren con mayor frecuencia dolor crónico y se relata con mayor intensidad y duración en comparación con los hombres (Meeder et al., 2010). Además, algunos autores establecen que esta relación sería una combinación de variables biológicas, sicológicas y sociales (Phillips et al.).

Del total de individuos adultos mayores que presentaron algún diagnóstico de TTM, los desórdenes articulares (TTM Grupo II) fueron los más prevalentes en ambos sexos, con un $33 \%$ de prevalencia general, con el diagnóstico de desplazamiento discal con reducción. Al contrario de lo pensado por el grupo, donde se esperaba una mayor prevalencia de diagnósticos del tipo inflamatorio (Grupo III). Esto podría ser explicado, por los criterios de exclusión establecidos en la metodología del estudio, donde se indica que no fue incluido ningún sujeto que tuviera previamente antecedentes de artrosis, artritis o lupus (Dworkin \& LeResche).

Respecto a la necesidad de tratamiento de TTM en la población adulta mayor, se estimó que el $30 \%$ requeriría de atención por la disfunción detectada. Los diagnósticos establecidos, se caracterizaban por tener sintomatología dolorosa en la musculatura asociada (dolor miofascial; grupo la) o en la articulación (artritis; grupo IIla). Sin embargo, es necesario realizar más estudios, donde se incluya el aspecto psicológico del paciente (Eje II de Dworkin), ya que la depresión, la ansiedad y la fatiga, en conjunto, determinan un mayor riesgo de que el dolor agudo relacionado con los TTM se convierta en crónico y que además los signos relacionados con los TTM se perpetúen (Espinosa et al., 2009) lo que en última instancia podría complicar más el tratamiento.

SANDOVAL, I.; IBARRA, N.; FLORES, G.; MARINKOVIC, K.; DÍAZ, W. \& ROMO, F. Prevalence of temporomandibular disorders according to RDC/TMD in older people of Santiago, Chile. Int. J. Odontostomat., 9(1):73-78, 2015.

ABSTRACT: In Chilean population, elders represent a 13\% of the overall population (2.2 million), projected for 2020 an increase of $45 \%$, from the group of 60 and over. All humans undergo a process of aging. Oral and perioral tissues do not escape from this process, whose impact on the condyle and articular disc could be observed, in the presence of symptoms consistent with temporomandibular disorders (TMD). The aim of this study was to determine the prevalence of TMD according to the Research Diagnostic Criteria or RDC/TMD (Axis I), in older adults examined at the Dental Clinic of the Faculty of Dentistry, University of Chile during 2012, and establish the relationship between the sexes. In conclusion, this study found that there is a high prevalence of TMD in Chilean elderly sample studied (47\%), mainly articular diagnoses without a statistically significant relationship between the sexes.

KEY WORDS: elders, temporomandibular disorders, RDC/TMD. 


\section{REFERENCIAS BIBLIOGRÁFICAS}

Corsini, G.; Fuentes, R.; Bustos, L.; Borie, E.; Navarrette, A.; Navarrete, D. \& Fulgeri, B. Temporomandibular disorders signs and symtoms determination of 13 to 18 years old students from a school in Temuco, Chile. Int. J. Morphol., 23(4):345-52, 2005.

Drummond, J.; Newton, J. \& Scott, J. Orofacial ageing. In: Barnes, I. E. \& Walls, A. (Eds.). Gerodontology. Oxford, Wright, 1994. pp.17:28.

Dworkin, S. F.; Huggins, K. H.; LeResche, L.; Von Korff, M.; Howard, J.; Truelove, E. \& Sommers, E. Epidemiology of signs and symptoms in temporomandibular disorders: clinical signs in cases and controls. J. Am. Dent. Assoc., 120(3):273-81, 1990.

Dworkin, S. F. \& LeResche, L. Research diagnostic criteria for temporomandibular disorders: review, criteria, examinations and specifications, critique. J. Craniomandib. Disord., 6(4):301-55, 1992.

Espinosa, S. I.; Lara, M. C.; Lara, C. A.; Saavedra, G. M. \& Vargas, G. H. Comparación de los aspectos psicosociales (eje II) de los pacientes con trastornos temporomandibulares, de acuerdo a la combinación de diagnósticos físicos (eje I) de los criterios diagnósticos para la investigación de los trastornos temporomandibulares (CDI/TTM). Oral, 10(30):477-81, 2009.

Grau, L. I.; Fernández, L. K.; González, G. \& Osorio, N. M. Algunas consideraciones sobre los trastornos temporomandibulares. Rev. Cuba. Estomatol., 42(3), 2005. Disponible en: http://scielo.sld.cu/ scielo.php? script=sci_arttext\&pid=S0034$75072005000300005 \&$ Ing $=e s$

Griffiths, R. H. Report of the President's conference on the examination, diagnosis, and management of temporomandibular disorders. Am. J. Orthod. Dentofac. Orthop., 83(6):514-7, 1983.

Instituto Nacional de Estadísticas (INE). Población adulta mayor en el bicentenario. Enfoque Estadístico. En: Instituto Nacional de Estadísticas (INE). Boletín Informativo del Instituto Nacional de Estadísticas. Santiago de Chile, Subdepartamento de Estadísticas Demográficas, Instituto Nacional de Estadísticas (INE), 2010.

Laskin, D. M. Etiology of the pain-dysfunction syndrome. J. Am. Dent. Assoc., 79(1):147-53, 1969.

Lee, H. C.; TI Huang, K. \& Shen, W. K. Use of antiarrhythmic drugs in elderly patients. J. Geriatr. Cardiol., 8(3):184-94, 2011.

Leresche, L. \& Drangsholt, M. Epidemiology of orofacial pain: prevalence, incidence, and risk factors. In: Sessle, B. J.; Lavigne, G. J.; Lund, J. P. \& Dubner, R. (Eds.). Orofacial pain. From basic science to clinical management. 2a ed. Chicago, Quintessence Publishing, 2008. pp.13-8.

Levy, B. Disease-related changes in older adults. In: Papas, A.; Niessen, L. \& Chauncey, H. (Eds.). Geriatric Dentistry. Aging and Oral health. St. Louis, Mosby, 1991. pp.83-102.

Meeder, B. W.; Weiss, V. F.; Maulén, Y. M.; Lira, A. D.; Padilla, L. G. R.; Hormazábal, N. F. \& Guerrero, M. L. Trastornos temporomandibulares: Perfil clínico, comorbilidad, asociaciones etiológicas y orientaciones terapéuticas. Av. Odontoestomatol., 26(4):209-16, 2010.
Ministerio de Planificación (MIDEPLAN). Documento № 12 Situación de los adultos mayores en Chile. En: Ministerio de Planificación (MIDEPLAN). Resultados de la VII Encuesta de Caracterización Socioeconómica Nacional (CASEN 2000). Santiado de Chile, Ministerio de Planificación (MIDEPLAN), 2000. Disponible en: http:/ / w w w. e s e.ualg.pt/gerontolog i a / Art\%C3\%ADculos\%20espa\%C3\%B1ol/CASEN\%202000.pdf

Ministerio de Salud (MINSAL). Diagnóstico de Situación de Salud Bucal. Santiago de Chile, Ministerio de Salud (MINSAL), 2010. Disponible en: http://web.minsal.cl/portal/url/item/ 7dc33df0bb34ec58e04001011e011c36.pdf

Misrachi, C. M.; Cabargas, J. \& Acevedo, X. Salud oral en el adulto mayor. Santiago de Chile, Lora Impresiones, 2005.

Ministerio de la Mujer y Desarrollo Social de Perú. Instituto Nacional de Bienestar Familiar. Autocuidado de la salud para el adulto mayor: manual de información para profesionales. Lima, MIMDES, 2003. pp.19-31.

Okeson, J. P. Tratamiento de oclusión y afecciones temporomandibulares. $5^{\text {a }}$ ed. Madrid, Elsevier Mosby, 2003. pp.149-233.

Petersen, P. E. \& Yamamoto, T. Improving the oral health of older people: the approach of the WHO Global Oral Health Programme. Community Dent. Oral Epidemiol., 33(2):81-92, 2005.

Phillips, J. M.; Gatchel, R. J.; Wesley, A. L. \& Ellis, E. 3rd. Clinical implications of sex in acute temporomandibular disorders. J. Am. Dent. Assoc., 132(1):49-57, 2001.

Pino, C. Manual de Trastornos Temporomandibulares. Santiago de Chile, Curso de Formación Avanzada en Oclusión. Facultad de Odontología de la Universidad de Chile, Escuela de postgrado, 2001.

Scully, C. \& Ettinger, R. L. The influence of systemic diseases on oral health care in older adults. J. Am. Dent. Assoc., 138(Suppl.):7S14S, 2007.

Taboada, A. O.; Gómez, G. Y. L.; Taboada, A. S. \& Mendoza, N. V. M. Prevalencia de signos y síntomas de los trastornos temporomandibulares en un grupo de adultos mayores. Rev. Asoc. Dent. Mex., 61(4):125-9, 2004

Yap, A. U.; Dworkin, S. F.; Chua, E. K.; List, T.; Tan, K. B. \& Tan, H. H. Prevalence of temporomandibular disorder subtypes, psychologic distress, and psychosocial dysfunction in Asian patients. J. Orofac. Pain, 17(1):21-8, 2003.

Dirección para Correspondencia:

Prof. Dr. Walter Díaz Guzmán

Especialista en TTM y DOF

Clínica de Odontología Integral del Adulto

Departamento de Prótesis

Universidad de Chile

Santiago

CHILE

Email: walterdiazg@gmail.com

Recibido : 20-06-2014

Aceptado: 14-01-2015 